\title{
Exploring self-report and proxy-report quality-of-life measures for people living with dementia in care homes
}

\author{
Alys W. Griffiths ${ }^{1}$ (I) Sarah J. Smith ${ }^{1} \cdot$ Adam Martin $^{2} \cdot$ David Meads $^{2} \cdot$ Rachael Kelley $^{1} \cdot$ Claire A. Surr $^{1}$
}

Accepted: 11 October 2019 / Published online: 23 October 2019

(c) The Author(s) 2019

\begin{abstract}
Purpose There are many validated quality-of-life (QoL) measures designed for people living with dementia. However, the majority of these are completed via proxy-report, despite indications from community-based studies that consistency between proxy-reporting and self-reporting is limited. The aim of this study was to understand the relationship between self- and proxy-reporting of one generic and three disease-specific quality-of-life measures in people living with dementia in care home settings.

Methods As part of a randomised controlled trial, four quality-of-life measures (DEMQOL, EQ-5D-5L, QOL-AD and QUALID) were completed by people living with dementia, their friends or relatives or care staff proxies. Data were collected from 726 people living with dementia living in 50 care homes within England. Analyses were conducted to establish the internal consistency of each measure, and inter-rater reliability and correlation between the measures.

Results Residents rated their quality of life higher than both relatives and staff on the EQ-5D-5L. The magnitude of correlations varied greatly, with the strongest correlations between EQ-5D-5L relative proxy and staff proxy. Internal consistency varied greatly between measures, although they seemed to be stable across types of participants. There was poor-to-fair inter-rater reliability on all measures between the different raters.

Discussion There are large differences in how QoL is rated by people living with dementia, their relatives and care staff. These inconsistencies need to be considered when selecting measures and reporters within dementia research.
\end{abstract}

Keywords Proxy-report $\cdot$ Care homes $\cdot$ Dementia $\cdot$ Inter-rater reliability

At least $70 \%$ of care home residents in the UK [1] and over $50 \%$ of nursing home residents in the USA live with dementia [2]. As over half of residents die within 1 year of moving into a care home [3] and there is currently no cure for dementia, ensuring that individuals living with the condition maintain their quality of life is a priority within care homes. Quality of life (QoL) is often a key outcome measure in research studies evaluating the effectiveness of interventions in care home settings [4]. Additionally, changes in QoL have been examined as an indicator of the progression of dementia; studies show residents with a higher dependency on staff generally have a lower quality of life [5]. Measuring QoL

$\triangle$ Alys W. Griffiths

alys.griffiths@leedsbeckett.ac.uk

1 Centre for Dementia Research, Leeds Beckett University, Leeds LS1 3HE, UK

2 Academic Unit of Health Economics, Leeds Institute of Health Sciences, University of Leeds, Leeds LS2 9JT, UK has multiple important uses for clinical practice and research in care home settings; it is therefore critical to have accurate and validated tools to measure it in people living with dementia.

QoL is a subjective construct and participants usually rate their own experiences using self-report outcome measures. People living with dementia in care settings may have difficulties with communication, reasoning and recall accuracy [6], thus proxy informant outcome measures are commonly used alongside, or instead of, self-rated measures [7]. However, the use of proxy measures raises several issues with regard to accuracy. It is well established that proxy raters assess QoL lower than self-rated QoL within dementia research [8], since these two groups may have different concepts of QoL [9]. Correlation between scores on QoL measures specifically designed for people with dementia are generally low-to-moderate between self-, staff-proxy and relative-proxy, suggesting poor agreement $[10,11]$ and it is unclear who is the more accurate reporter [12]. The 
difference between proxy- and self-rated QoL is greater for individuals with higher levels of impaired cognition [10]. These proxies also rate the QoL of people with dementia as lower when they (the person with dementia) are experiencing more distress [10]. Evidence demonstrates that some people with dementia may overestimate their quality of life, suggesting a ceiling effect [8]. For example within one study, almost $50 \%$ of residents rated themselves as having the highest possible quality of life [12]. An additional issue for intervention research is the inability to blind proxies to treatment allocation in some studies, particularly when evaluating psychosocial interventions. Furthermore, the relationship between the proxy and resident, as well as time spent with the person during the reporting period, may influence proxy ratings [13]. These factors may affect measurement accuracy, increasing the chance of reporting errors. Despite these issues, there has been little examination of the relationship between different proxy raters across multiple measures.

Clinical trials rely on accurate outcome measurements and therefore, frequently collect data using multiple measures and multiple raters. A wide range of QoL measures exist for use with individuals with dementia, disease-specific (i.e. QUALID, DEMQoL) and generic (i.e. EQ-5D-5L). There are differences in these measures, in terms of their conceptualisation of QoL and procedures around administration or scoring [14]. Additionally, there is no recommended or standardised set of QoL measures for use in clinical trials of psychosocial interventions. One recent review identified five different dementia-specific QoL measures used by clinical trials in the past 10 years [15], making comparison of results between trials difficult. To address measurement issues and potential bias, researchers often choose to use more than one QoL measure within a research project [16], typically utilising a combination of self-rated and proxyrated measures as appropriate.

Despite the widespread use of various QoL measures in dementia research, there has been limited comparison between self-report and staff proxy measurements in people living with dementia in care homes, where proxy-reporting is often relied upon [17]. Generally, low agreement between self-reported and staff proxy-reported QoL has been found, with mean resident-reported scores on the EQ-5D-5L [11] and QOL-AD [18] higher than those of staff proxies. The current evidence base, however, provides limited evidence of how ratings vary between different proxy-reporters and different measures, or how measures capture changes in QoL over time. To date, psychometric evaluation has usually examined differences between raters on a single QoL measure, limiting understanding of which QoL measures might be most appropriate, or how different measures compare for a single rater. Consequently, there is little consensus around the optimal way to measure QoL for people living with dementia [18]. Given QoL is one of the most frequent outcome measures used in dementia-related clinical trials, research on the relationship between measures is required to support selection of the most appropriate outcomes and raters, potentially leading to increased quality in outcome measurement.

To address this issue, this study examined aspects of validity and reliability, and relationships between four $\mathrm{QoL}$ measures across a large care home-based sample of three different raters (self-, staff proxy- and relative proxy-reporters). The aim of the study was to demonstrate how these differ, to allow researchers to consider which measure(s) and rater(s) is/are most appropriate for their research questions.

\section{Method}

\section{Participants and procedure}

Participants living with dementia were recruited from 50 care homes as part of a randomised controlled trial (for further details see [19]). Residents were eligible to participate if they lived in the care home permanently (i.e. were not receiving respite care), had a formal diagnosis of dementia or scored $\geq 4$ on the Functional Assessment Staging of Alzheimer's disease (FAST) [20]. They were ineligible if they had been formally admitted to an end-of-life care pathway or were mainly cared for in bed. For each participant, a staff proxy was recruited, who was eligible if he/she knew the resident well and had a permanent contract with the care home. The staff proxy was usually the resident's key worker (i.e. a member of care staff). Where possible, a relative or friend of each person living with dementia was also recruited. The only eligibility criterion for relatives and friends was that they visited at least once every 2 weeks. All participants required sufficient proficiency in English to complete the measures.

Ethical approval was granted by the Bradford Leeds National Research Ethics Service Committee and Leeds Beckett University research ethics committee.

As part of the trial data collection, four QoL outcome measures were completed for each resident. Data collection took place over a two-week period. Proxy-reporters were asked to complete measures reflecting on QoL either 'today' or over 'the past 2 weeks' dependent on measure instructions; therefore, if they had not spent time with the person with dementia during this time, the research team sought another proxy. Some participants with dementia did not complete all measures, most frequently due to feeling too tired to continue. 


\section{Quality-of-life measures}

\section{EQ-5D-5L [21]}

The EQ-5D-5L is a five-item general (non-disease-specific) QoL measure that covers five dimensions: usual activities, mobility, self-care, anxiety/depression and pain. Respondents rate each item in terms of the level of problem they have with this domain (no problems, slight problems, moderate problems, severe problems and unable to complete task). An index score is calculated, from -0.281 to 1 , where higher scores indicate higher QoL, using health state valuations provided by country-specific general populations. This measure was completed by people living with dementia, staff proxy-reporters and relative/friend proxy-reporters. The EQ-5D-5L has been used with people with mild-to-severe dementia; however, there are concerns about its validity amongst this latter population [12].

\section{DEMQOL proxy [22]}

The DEMQOL proxy is a disease-specific QoL measure for people with dementia. It consists of 32 items that measures six domains of general health, mood, behavioural symptoms, cognition and memory, and physical and social functioning. Items are rated on a four-point Likert scale ranging from 'a lot' to 'not at all', with higher scores indicating higher QoL (five items are reverse scored). Scores range from 31 to 124 . This measure was completed by staff proxy-reporters and relative proxy-reporters.

\section{QOL-AD Nursing Home [23]}

The QOL-AD Nursing Home version is a disease-specific 15-item questionnaire designed to measure QoL for people living with dementia in care homes. It covers areas including mood, relationship with friends and family, and physical condition. There are wording changes from the original QOL-AD to increase the relevance of the measure to people living in care homes, such as removal of an item around marital status and the addition of items related to relationships with staff and ability to make choice in daily life. Completed via self-report, this questionnaire uses simple language with four response options that are consistent across all items (poor, fair, good or excellent). Items are rated on a four-point Likert scale, with higher scores indicating higher QoL. Scores range from 15 to 60 . Residents with mild-to-moderate dementia are reported to be able to self-rate QoL using this measure [18].
Qualid [24]

The QUALID is a disease-specific 11-item proxy-rated measure that rates both the presence and the frequency of indicators of QoL during the past 7 days. The measure covers 11 behavioural areas thought indicative of positive and negative QoL. A five-point Likert scale captures the frequency of each item, with total scores ranging from 11 to 55 , with higher scores indicating higher QoL. This measure was completed by staff and relative proxy-reporters.

\section{Measure of functioning}

\section{FAST [20]}

The Functional Assessment Staging of Alzheimer's disease (FAST) measures the functional severity of dementia and was completed by a researcher with the care home manager. The tool is rated from 1 (no dementia) to 7 (severe dementia), with additional sublevels for 6 and 7 (a-e). To be eligible to participate in the present work, individuals were required to have a FAST score of 4 or above. This tool was completed to ensure that those without a formal diagnosis but who were still eligible could be recruited and was used to provide an understanding of sample demographics.

\section{Missing data}

A researcher completed measures with each participant (except for some relative/friend proxy measures that were completed via post); therefore, the frequency of missing data at the participant level was extremely low, less than $1 \%$ for any one participant measure. Where some items were missing from a measure, this was dealt with by imputing the participant-specific mean item score in line with guidance [25]. Where a measure was not completed at all, this was marked as missing and not included in analyses. Therefore, different numbers of participants completed each measure and is highlighted where relevant.

\section{Data analysis}

Data were analysed using SPSS v24. Correlations between measures were conducted to investigate concurrent validity, and correlations between assessors were conducted to establish inter-rater reliability. Spearman's correlations were conducted between each of the measures, for self-report, staff proxy and relative/friend proxy, to establish whether significant correlations existed between the measures, and the magnitude of these. To calculate the internal consistency of measures, Cronbach's alpha was conducted.

Inter-rater reliability was conducted between rater type on each of the QoL measures that were completed by at least 
two raters, using the weighted Cohen's Kappa statistic with linear weights. The strength of the relationship was investigated to establish the level of agreement between raters over and above chance (ranging from -1 to +1 ) based on guidance [26]. The strength of the relationship is represented in the Cohen's Kappa statistic as follows: values $\leq 0$ indicate no agreement, $0.01-0.20$ as poor agreement, $0.21-0.40$ as fair, $0.41-0.60$ as moderate, $0.61-0.80$ as good and $0.81-1.00$ as almost perfect agreement.

\section{Results}

A total of 726 resident participants were recruited (see Table 1 for demographics) from 50 care homes, of which 377 completed self-report measures. Most participants were female $(536 ; 74 \%)$, identified as White British $(702 ; 96 \%)$ and had an average age of 85 (range 57-102). A staff proxy

Table 1 Participant demographics

\begin{tabular}{ll}
\hline Characteristics & \\
\hline Age at registration (years) M(SD) & $85.6(7.64)$ \\
Gender & \\
Female & $536(73.8 \%)$ \\
Male & $190(26.2 \%)$ \\
Length of stay in care home (years) M(SD) & $2.3(2.34)$ \\
Ethnicity & \\
White British/European & $702(96.7 \%)$ \\
Other & $24(3.3 \%)$ \\
Funding type & \\
Local authority & $352(48.5 \%)$ \\
Self-funded & $289(39.8 \%)$ \\
Local authority and self-funded & $34(4.7 \%)$ \\
Continuing Healthcare & $48(6.6 \%)$ \\
Missing & $3(0.4 \%)$ \\
FAST & \\
1-3 & $6(1 \%)$ \\
4 & $95(13.6 \%)$ \\
5 & $74(10.6 \%)$ \\
6 & $380(54.5 \%)$ \\
7 & $142(20.4 \%)$ \\
Missing & $29(3.9 \%)$ \\
QoL measures (baseline scores) & $\mathrm{M}(\mathrm{SD}) \mathrm{N}$ \\
EQ-5D-5L (self-report) & $.868(.18) 365$ \\
EQ-ED-5L (staff proxy) & $.668(.23) 723$ \\
EQ-ED-5L (relative proxy) & $.520(.25) 162$ \\
QUALID (staff proxy) & $20.5(6.95) 726$ \\
QUALID (relative proxy) & $22.0(7.18) 163$ \\
QOL-AD (self-report) & $42.54(6.31) 344$ \\
DEMQOL (staff proxy) & $104.16(9.77) 714$ \\
DEMQOL (relative proxy) & $98.93(14.30) 152$ \\
\hline
\end{tabular}

was recruited for each individual alongside 197 relatives/ friend proxies.

\section{Correlation between measures}

There were significant correlations between most measures across different reporters (see Table 2). For relative proxycompleted measures, QUALID correlated with all other measures, EQ-5D-5L correlated with all measures except DEMQOL staff proxy and DEMQOL correlated with all but one (EQ-5D-5L staff proxy) measures. For self-report measures, EQ-5D-5L correlated with all other measures and QOL-AD correlated with all self-report and relative proxy-completed measures, but only QUALID of the staff proxy-completed measures. The magnitudes of these correlations varied, with the strongest correlations between EQ-5D-5L relative proxy with EQ-5D-5L staff proxy (.60) and self-report (.45), and QUALID staff (.42) and relative (.48) proxies.

There were a greater number of staff and relative proxy ratings overall, potentially as a function of scores not being completed by residents with more severe cognitive impairment. To explore whether this impacted the pattern of findings, mean staff and relative proxy ratings for EQ-5D-5L index scores were compared only for residents who provided an equivalent self-rating. When both scores related to the same group of residents, self-report scores (.78) remained higher than staff proxy-completed scores (.53), and selfreport scores (.69) also remained higher than relative/friend proxy-completed scores (.41).

\section{Internal consistency}

Internal consistency varied greatly between measures, although it seemed to be stable across types of participants (see Table 3). The DEMQOL (staff and relative/friend proxies) had good to excellent internal consistency (0.8-1.0), the QOL-AD (self-report) had good internal consistency (0.8-0.9), the QUALID (staff and relative/friend proxies) had acceptable internal consistency (0.7-0.8) and ED-5D-5L (all participants) had questionable internal consistency (0.6-0.7) [27].

\section{Inter-rater reliability}

Agreement between the staff proxy-rated $(M=22.46)$ and relative/friend proxy-rated $(M=22.18)$ QUALID ratings $(N=159)$ indicated that although the level of agreement was above chance, there was a fair level of agreement between raters $(k=.306 p<.001$; see Table 4). Agreement between staff proxy $(M=101.99)$ and relative/friend proxy $(M=$ 98.97) DEMQoL ratings $(N=150)$ was similarly above 
Table 2 Correlations between measures $-N$ participants provided in brackets

\begin{tabular}{|c|c|c|c|c|c|c|c|c|}
\hline & $\begin{array}{l}\text { EQ-5D-5L } \\
\text { (self-report) }\end{array}$ & $\begin{array}{l}\text { EQ-5D-5L } \\
\text { (staff proxy) }\end{array}$ & $\begin{array}{l}\text { EQ-5D-5L } \\
\text { (relative } \\
\text { proxy) }\end{array}$ & $\begin{array}{l}\text { QUALID } \\
\text { (staff } \\
\text { proxy) }\end{array}$ & $\begin{array}{l}\text { QUALID } \\
\text { (relative } \\
\text { proxy) }\end{array}$ & $\begin{array}{l}\text { QOL-AD } \\
\text { (self- } \\
\text { report) }\end{array}$ & $\begin{array}{l}\text { DEMQOL } \\
\text { (staff proxy) }\end{array}$ & $\begin{array}{l}\text { DEMQOL } \\
\text { (relative } \\
\text { proxy) }\end{array}$ \\
\hline EQ-5D-5L (self-report) & - & $\begin{array}{l}.18^{* *} \\
(377)\end{array}$ & $\begin{array}{l}.45^{* *} \\
(81)\end{array}$ & $\begin{array}{l}.11^{*} \\
(377)\end{array}$ & $\begin{array}{l}.33 * * \\
(79)\end{array}$ & $\begin{array}{l}.30 * * \\
(329)\end{array}$ & $\begin{array}{l}.12 * \\
(372)\end{array}$ & $\begin{array}{l}.39 * * \\
(75)\end{array}$ \\
\hline EQ-5D-5L (staff proxy) & $\begin{array}{l}.18^{* *} \\
(377)\end{array}$ & - & $\begin{array}{l}.60^{* *} \\
(166)\end{array}$ & $\begin{array}{l}.33^{* *} \\
(691)\end{array}$ & $\begin{array}{l}.27 * * \\
(164)\end{array}$ & $\begin{array}{l}.09 \\
(336)\end{array}$ & $\begin{array}{l}.11^{*} \\
(713)\end{array}$ & $\begin{array}{l}.05 \\
(152)\end{array}$ \\
\hline EQ-5D-5L (relative proxy) & $\begin{array}{l}.45^{* *} \\
(81)\end{array}$ & $\begin{array}{l}.60^{* *} \\
(166)\end{array}$ & - & $\begin{array}{l}.42 * * \\
(160)\end{array}$ & $\begin{array}{l}.48 * * \\
(162)\end{array}$ & $\begin{array}{l}.31 * \\
(68)\end{array}$ & $\begin{array}{l}.07 \\
(164)\end{array}$ & $\begin{array}{l}.29 * * \\
(150)\end{array}$ \\
\hline QUALID (staff proxy) & $\begin{array}{l}.11^{*} \\
(377)\end{array}$ & $\begin{array}{l}.33^{* *} \\
(691)\end{array}$ & $\begin{array}{l}.42 * * \\
(160)\end{array}$ & - & $\begin{array}{l}.50 * * \\
(159)\end{array}$ & $\begin{array}{l}.11 * \\
(325)\end{array}$ & $\begin{array}{l}.48 * * \\
(680)\end{array}$ & $\begin{array}{l}.20 * \\
(148)\end{array}$ \\
\hline QUALID (relative proxy) & $\begin{array}{l}.33^{* *} \\
(79)\end{array}$ & $\begin{array}{l}.27 * * \\
(164)\end{array}$ & $\begin{array}{l}.47 * * \\
(162)\end{array}$ & $\begin{array}{l}.50 * * \\
(159)\end{array}$ & - & $\begin{array}{l}.28 * \\
(67)\end{array}$ & $\begin{array}{l}.28 * * \\
(162)\end{array}$ & $\begin{array}{l}.51^{* *} \\
(150)\end{array}$ \\
\hline QOL-AD (self-report) & $\begin{array}{l}.30 * * \\
(329)\end{array}$ & $\begin{array}{l}.09 \\
(336)\end{array}$ & $\begin{array}{l}.31 * \\
(68)\end{array}$ & $\begin{array}{l}.11 * \\
(325)\end{array}$ & $\begin{array}{l}.28 * \\
(67)\end{array}$ & - & $\begin{array}{l}.08 \\
(332)\end{array}$ & $\begin{array}{l}.42 * * \\
(63)\end{array}$ \\
\hline DEMQOL (staff proxy) & $\begin{array}{l}.12^{*} \\
(372)\end{array}$ & $\begin{array}{l}.11 * \\
(713)\end{array}$ & $\begin{array}{l}.07 \\
(164)\end{array}$ & $\begin{array}{l}.48 * * \\
(680)\end{array}$ & $\begin{array}{l}.28 * * \\
(162)\end{array}$ & $\begin{array}{l}.08 \\
(332)\end{array}$ & - & $\begin{array}{l}.37 * * \\
(150)\end{array}$ \\
\hline DEMQOL (relative proxy) & $\begin{array}{l}.39 * * \\
(75)\end{array}$ & $\begin{array}{l}.05 \\
(152)\end{array}$ & $\begin{array}{l}.29 * * \\
(150)\end{array}$ & $\begin{array}{l}.20^{*} \\
(148)\end{array}$ & $\begin{array}{l}.51^{* *} \\
(150)\end{array}$ & $\begin{array}{l}.42 * * \\
(63)\end{array}$ & $\begin{array}{l}.37 * * \\
(150)\end{array}$ & - \\
\hline
\end{tabular}

$* p<.05, * * p<.001$

Table 3 Cronbach's alpha values for self- and proxy-completed measures by type of participant

\begin{tabular}{llll}
\hline & Self-report & Staff proxy & Relative proxy \\
\hline EQ-5D-5L & .69 & .62 & .67 \\
QUALID & - & .74 & .72 \\
QOL-AD & .86 & - & - \\
DEMQOL proxy & - & .83 & .91 \\
\hline
\end{tabular}

chance with poor/fair level of agreement between raters $(k=.205, p<.001)$.

Agreement between ratings on the EQ-5D-5L descriptive and Index scores was also explored. There was fair agreement between staff proxy (11.47) and relative/friend proxy (14.14) ratings $(n=166)$ on the descriptive scale $(k=.323$ $p<.0005)$. However, there was poor agreement between the 377 cases of staff proxy (10.22) and resident (7.46) ratings $(k=.121 p<.0005)$. Similarly, in the 80 cases where relative/friend proxies (13.80) and residents (8.64) completed the EQ-5D-5L, there was poor agreement between ratings $(k=.170 p<.0005)$.

Table 4 Inter-rater reliability within QoL measures: Cohen's Kappa

\begin{tabular}{|c|c|c|c|c|c|c|c|c|}
\hline & $\begin{array}{l}\text { EQ-5D-5L } \\
\text { (self-report) } \\
\text { Desc. }\end{array}$ & $\begin{array}{l}\text { EQ-5D-5L } \\
\text { (self-report) } \\
\text { Ind. }\end{array}$ & $\begin{array}{l}\text { EQ-5D-5L } \\
\text { (staff proxy) } \\
\text { Desc. }\end{array}$ & $\begin{array}{l}\text { EQ-5D-5L } \\
\text { (staff proxy) } \\
\text { Ind. }\end{array}$ & $\begin{array}{l}\text { EQ-5D-5L } \\
\text { (relative proxy) } \\
\text { Desc. }\end{array}$ & $\begin{array}{l}\text { EQ-5D-5L } \\
\text { (relative proxy) } \\
\text { Ind. }\end{array}$ & $\begin{array}{l}\text { QUALID } \\
\text { (relative } \\
\text { proxy) }\end{array}$ & $\begin{array}{l}\text { DEMQOL } \\
\text { (relative } \\
\text { proxy) }\end{array}$ \\
\hline $\begin{array}{l}\text { EQ-5D-5L (self- } \\
\text { report) Desc. }\end{array}$ & & & $.121 * *$ & & $.170 * *$ & & & \\
\hline $\begin{array}{l}\text { EQ-5D-5L (self- } \\
\text { report) Ind. }\end{array}$ & & & & .004 & & $.040 * *$ & & \\
\hline $\begin{array}{l}\text { EQ-5D-5L (staff } \\
\text { proxy) }\end{array}$ & & & & & $.323 * *$ & & & \\
\hline Desc. & & & & & & & & \\
\hline $\begin{array}{l}\text { EQ-5D-5L (staff } \\
\text { proxy) Ind. }\end{array}$ & & & & & & $.030 * *$ & & \\
\hline $\begin{array}{l}\text { QUALID (staff } \\
\text { proxy) }\end{array}$ & & & & & & & $.306^{*}$ & \\
\hline $\begin{array}{l}\text { DEMQOL (staff } \\
\text { proxy) }\end{array}$ & & & & & & & & $.205^{*}$ \\
\hline
\end{tabular}


Agreement between the Index EQ-5D-5L ratings was computed using the Cohen's $k$ statistic. As with the descriptive score, there was low agreement between resident and relative/friend ratings $k=.04 p<.0005$. There was very low agreement between resident and staff proxy ratings of Index QoL that was not statistically different to chance $(k=.004$ $p=.649)$ and there was low agreement between the staff and relative/friend proxy ratings $(k=.030 p<.0005)$.

Additionally, examination of the EQ-5D-5L by domain was conducted, to establish where differences between raters existed. Given this is a 5-item measure, large discrepancies between raters on a single item produce larger impacts on the overall score than for measures with more items. This demonstrated that on all domains, residents rated themselves as having 'no problems' more frequently than either relative/ friend proxies or staff proxies (see Table 5). However, the difference was particularly large for self-care, where $76 \%$ of residents stated they had no problems with this whereas staff and relative/friend proxies rated that a much lower percentage of people were with no problems in this area (14\% and $10 \%$, respectively).

\section{Discussion}

The present study compared QoL measures for people living with dementia across multiple measures with multiple raters. The inclusion of three raters allowed comparisons to be drawn between three groups of participants who may be recruited for clinical trials where QoL is an outcome. The internal consistency of measures varied from questionable (EQ-5D-5L) to good and excellent (DEMQOL), in line with previous evidence [see 28 for review]. The QOL-AD NH has been shown to have variable internal consistency [29,
30]; in the present study, however, we found the measure to have good internal consistency. This may be due to differing levels of cognitive impairment between samples, which may affect how reliably measures are completed. For example, a small sample of individuals with mild dementia was recruited for one study where the scale demonstrated good internal consistency [30], whereas a second study excluded those with advanced dementia [29]. Therefore, further research is required to establish when use of the QOL-AD $\mathrm{NH}$ is appropriate.

Correlations between different measures across different reporters were generally weak to moderate, in line with recent similar studies $[11,31]$. This suggests that people living with dementia and those who support them do not perceive QoL in the same way, or that they may focus on different aspects of QoL, suggesting a need for several measures to be completed to ensure full coverage of perceived QoL. However, the issues may instead be due to differences in how QoL is conceptualised by people with dementia and by different types of proxy informant. This is especially important as proxy raters are thought to focus on issues such as pain and presence of neuropsychiatric symptoms, rather than QoL specifically [18]. Recent qualitative research suggests that staff members equate residents' QoL with the quality of care delivered or the stage of their dementia, whereas relatives draw comparisons with the person's QoL when they were younger, lived in their own home and did not have dementia [31]. It is unclear how people with dementia, particularly those who are care home residents, conceptualise their QoL compared to proxies, although those who are experiencing pain and have recently had a fall report lower QoL [18]. Future qualitative work should be undertaken to understand how QoL is conceptualised and reflected on by different types of participants when completing these measures.
Table 5 Percentage of participants identifying problems or no problems across EQ-5D domains $[N(\%)]$

\begin{tabular}{lccc}
\hline EQ-5D dimension & Residents $(N=377)$ & Relative $(N=167)$ & Staff $(N=726)$ \\
\hline Mobility & & & \\
No problems & $248(66)$ & $31(19)$ & $457(37)$ \\
Problems & $129(34)$ & $136(81)$ & \\
Self-care & & & $100(14)$ \\
No problems & $285(76)$ & $16(10)$ & $626(86)$ \\
Problems & $92(24)$ & $151(90)$ & $461(64)$ \\
Usual activities & & & $265(36)$ \\
No problems & $307(81)$ & $41(25)$ & \\
Problems & $70(19)$ & $126(75)$ & $523(72)$ \\
Pain/discomfort & & & $203(28)$ \\
No problems & $271(72)$ & $77(46)$ & \\
Problems & $106(28)$ & $90(54)$ & $500(69)$ \\
Anxiety/depression & & $78(46)$ & $226(31)$ \\
No problems & $275(73)$ & $89(54)$ & \\
Problems & $102(27)$ &
\end{tabular}


Our findings broadly indicated an at best, fair agreement, between how the different raters perceived QoL for people living with dementia. The QUALID staff and relative/friend proxy ratings yielded the highest level of agreement, with fair agreement also found between staff and relative/friend proxies on the EQ-5D-5L. However, it is noted that fair agreement is not considered to be a reliable when establishing the validity of a measure, where a minimum value of .6 (substantial agreement) is recommended [32]. Notably, there was poor agreement between self-rated QoL and staff/relative proxy-rated QoL on the EQ-5D-5L. When the data were examined as Index values, agreement was not statistically above chance. This is in line with previous research, which has found discrepancies between people with dementia and their family members on this measure [33]. Further analyses, comparing the percentage of individuals who reported having problems in areas vs not having a problem in the area, revealed interesting discrepancies. Particularly, most residents reported no problems with self-care, whereas both staff and relative/friend proxies identified that most individuals had problems in this area. This may reflect additional issues with the sensitivity of this question, since people with dementia may feel uncomfortable or embarrassed stating they experience problems with self-care. Alternatively, care staff may overstate the problems individuals with dementia have, based on their own approach to provision of support for personal care, which may not be based on maximising independence, but rather on completing care tasks as efficiently as possible. Research should be conducted to explore these discrepancies in detail.

To date, a wealth of research studies have included multiple QoL and outcome measures but have not examined these systematically. For example, it has been highlighted that people with dementia are able to rate their QoL but that this differs from relative proxy ratings [34], without any exploration of why this might be. Other studies have stated that proxy ratings improve feasibility and should be used when people with dementia are unable to 'answer by themselves' to avoid having missing data [6], although this is presented without clear cut-offs to guide researchers. Therefore, researchers should be encouraged to examine the psychometric properties of the measures used within their studies, to help understand which are most appropriate for use with people living with dementia in care homes, with different degrees of cognitive impairment.

Previously, it has been stated that proxy-completed measures are the only option for individuals living with moderate to severe dementia $[6,9]$. However, this fails to value the perspective and the insights into the QoL of individuals of people with dementia that may not be picked up by staff members, relatives or friends. Additionally, for research findings and any resultant policy changes to be meaningful, appropriate and valid QoL data must be collected [10] including recognition that people with dementia are able to provide meaningful commentary around their own QoL [7]. Researchers should, therefore, explore creative ways to work with those who struggle to communicate verbally to collect meaningful data. The burden of data collection for people with dementia needs to be considered within this, as some participants were unable to complete measures in the present study due to tiredness or boredom. Flexibility in researcher approach has been highlighted as important, providing participants with the opportunity to complete measures through several conversations or over 2 days if required [35]. Building relationships with participants with dementia can help to identify the best time of day for data collection, which could help increase the feasibility of self-completed data [35]. Furthermore, people with dementia have been shown to consistently rate their QoL higher than proxy raters. It is unclear whether this relates to an inability to accurately assess their performance or abilities against measure items or whether in fact proxies under estimate QoL based on their own, different perceptions of what is important. For example, people with dementia living in a care home may compare themselves with others living in the setting and may judge their QoL to be good comparatively or they may have reduced expectations about their own performance given their personal circumstances or may simply have a more positive outlook [34]. It is also noted that people living with dementia may benefit from overestimating their QoL, as a strategy of self-maintenance [36]. Further research is thus needed to assess why people with dementia living in care home settings make particular judgements on QoL items and what this means for assumed 'accuracy' and interpretation of results.

More widely, there are concerns about the quality of QoL measures in general and the feasibility of their use with people with dementia. Most of the existing dementia-related QoL measures have had limited psychometric evaluation $[37,38]$. For example, to date, the relationship between the QOL-AD NH and any health-related outcomes has not been examined (criterion validity). Furthermore, the QUALID has demonstrated poor criterion validity, in both studies that have examined this $[39,40]$. However, whilst these issues are concerning, in part, these may be due to methodological issues of the studies examining the psychometric properties of measures rather than highlighting underlying problems with the measures [4].

\section{Limitations}

Although multiple measures were collected in the present study, only one measure was completed by residents that proxy-reporters also completed (EQ-5D-5L). Therefore, self- and proxy-rating comparisons could not be drawn 
from QUALID and DEMQOL. In future, where possible, the same measures should be completed by residents and proxy-reporters, in order to be able to draw more in-depth comparisons. Individuals who lived in the care home and were cared for in bed or were formally admitted to an end of life care pathway were not eligible to participate in the present study. These individuals may have been expected to have the lowest QoL and therefore the present study may not fully capture the breadth of QoL experienced by those living in care homes. Additionally, in line with previous evidence [9], those who completed self-report measures are likely to have been in the earlier stages of dementia and therefore have higher QoL than those in the later stages, which were not accounted for in the present study. Researchers need to develop alternative strategies to ensure that the perspectives of those with later-stage dementia are captured [35]. Furthermore, we do not understand why participants provided the rating that they did for each item. Collecting additional qualitative data to explore this issue would improve understanding around why differences in ratings exist.

There are demographic characteristics (for example in the qualifications and experience of staff, and relationships of relative/friends to residents) that may have affected QoL ratings [36]. For example, spouses have been found to rate QoL in people living with dementia as higher than adult children [33]. We did not stratify our analysis to explore within-group variations in QoL ratings due to poor completion of demographic details by these participants. Understanding predictors of variability in QoL ratings within relative/friend and staff proxy groups constitutes a valuable should be an ongoing focus for future research.

Future research should enhance recent reviews [37, 41] and conduct a meta-analysis of different QoL measures completed by different raters over time, in order to establish which are most meaningful and suitable for use. One recent narrative review concluded that self-report and proxy-report DEMQOL and EQ-5D-5L should be used [15]. However, within the 41 studies reviewed, only four used DEMQOL; therefore, this conclusion is based on limited evidence. We found that DEMQOL had good internal consistency in the present study and scores on this measure significantly correlated with five of the additional seven measures, although these correlations were all weak, except for staff proxyreported QUALID. In addition, QUALID correlated with all (relative/friend proxy-reporters) and all but one (staff proxy-reporters) measures. Although it demonstrated weaker internal consistency than both QOL-AD and DEMQOL, our results suggest that DEMQOL proxy may offer the most thorough and comparable measure of QoL; however, we did not collect self-report DEMQOL and cannot make a definitive judgment without this. A care home-specific version of the DEMQOL has recently been developed, in line with other measures such as the QOL-AD NH, which may provide further utility for this measure within care homes [42].

\section{Conclusion}

In conclusion, measuring quality of life for people with dementia is complex and often involves multiple measures completed by multiple raters. Whilst it is acknowledged that self-report data are the optimal method of data collection, the limitations of this method are also widely reported, particularly as ability to complete measures is likely to decline over time for those with dementia. The low levels of agreement between relative/friend and staff proxy raters on these measures, however, bring into question the appropriateness of proxy-rated data within this population. Given that residents may overestimate their QoL, it is difficult for researchers to establish which measure provides the most reliable or valid report of individuals' QoL. The lack of other viable alternatives at present means that researchers should be aware of these issues and interpret their data with caution. This study highlights the need for researchers and practitioners to better understand of the impact of rater choice on QoL outcomes. It is not possible to recommend staff or relative/friend proxy ratings as more or less accurate than self-ratings, as proxy rating may be biased by factors that unduly influence perceived QoL (such as self-care ability), whilst the same factors may have little impact on QoL as experienced by the participant or resident. Therefore, researchers need to give greater consideration of the influence of raters when selecting QoL outcome measures and their completion.

\section{Compliance with ethical standards}

Conflict of interest The authors declare no conflicts of interest

Ethical approval Ethical approval for the trial was granted by Leeds Bradford NRES Committee. The trial was registered with the International Standard Randomised Controlled Trial Register (ISRCTN) reference 82288852 . Approval for this substudy was granted by Leeds Beckett University ethics committee.

Informed consent All participants or where appropriate, a personal or nominated consultee, provided written informed consent prior to participation.

Open Access This article is distributed under the terms of the Creative Commons Attribution 4.0 International License (http://creativeco mmons.org/licenses/by/4.0/), which permits unrestricted use, distribution, and reproduction in any medium, provided you give appropriate credit to the original author(s) and the source, provide a link to the Creative Commons license, and indicate if changes were made. 


\section{References}

1. Prince, M., Knapp, M., Guerchet, M., McCrone, P., Prina, M., Comas-Herrera, A., et al. (2014). Dementia UK: Update (pp. 1-136). London: Alzheimer's Society.

2. Harris-Kojetin, L., Sengupta, M., Park-Lee, E., Valverde, R., Caffrey, C., Rome, V., et al. (2016). Long-term care providers and services users in the United States: Data from the National Study of Long-Term Care Providers, 2013-2014. Vital \& Health Statistics. Series 3, Analytical and Epidemiological Studies, 38, x-xii.

3. Kinley, J., Hockley, J. O., Stone, L., Dewey, M., Hansford, P., Stewart, R., et al. (2013). The provision of care for residents dying in UK nursing care homes. Age and Ageing, 43(3), 375-379.

4. Aspden, T., Bradshaw, S. A., Playford, E. D., \& Riazi, A. (2014). Quality-of-life measures for use within care homes: A systematic review of their measurement properties. Age and Ageing, 43, 596-603.

5. Jones, R. W., Romeo, R., Trigg, R., Knapp, M., Sato, A., King, D., et al. (2015). Dependence in Alzheimer's disease and service use costs, quality of life, and caregiver burden: the DADE study. Alzheimer's \& Dementia, 11(3), 280-290.

6. Diaz-Redondo, A., Rodriguez-Blazquez, C., Ayala, A., MartinezMartin, P., Forjaz, M. J., \& Spanish Research Group on Quality of Life and Aging. (2014). EQ-5D rated by proxy in institutionalized older adults with dementia: Psychometric pros and cons. Geriatrics \& gerontology international, 14(2), 346-353.

7. Moyle, W., Mcallister, M., Venturato, L., \& Adams, T. (2007). Quality of life and dementia: The voice of the person with dementia. Dementia, 6(2), 175-191.

8. Hounsome, N., Orrell, M., \& Edwards, R. T. (2011). EQ-5D as a quality of life measure in people with dementia and their carers: Evidence and key issues. Value in health, 14(2), 390-399.

9. Sheehan, B. D., Lall, R., Stinton, C., Mitchell, K., Gage, H., Holland, C., et al. (2012). Patient and proxy measurement of quality of life among general hospital in-patients with dementia. Aging \& Mental Health, 16(5), 603-607.

10. Robertson, S., Cooper, C., Hoe, J., Hamilton, O., Stringer, A., \& Livingston, G. (2017). Proxy rated quality of life of care home residents with dementia: A systematic review. International Psychogeriatrics, 29, 569-581.

11. Usman, A., Lewis, S., Hinsliff-Smith, K., Long, A., Housley, G., Jordan, J., et al. (2019). Measuring health-related quality of life of care home residents: Comparison of self-report with staff proxy responses. Age and Ageing, 27(1), 7-22.

12. Coucill, W., Bryan, S., Bentham, P., et al. (2001). EQ-5D in patients with dementia: an investigation of inter-rater agreement. Medical Care, 39(8), 760-771.

13. Gräske, J., Fischer, T., Kuhlmey, A., \& Wolf-Ostermann, K. (2012). Quality of life in dementia care-differences in quality of life measurements performed by residents with dementia and by nursing staff. Aging \& Mental Health, 16, 819-827.

14. Ettema, T. P., Droes, R. M., Lange, J. D., Mellenbergh, G. J., \& Ribbe, M. W. (2005). A review of quality of life instruments used in dementia. Quality of Life Research, 14, 675-686.

15. Yang, F., Dawes, P., Leroi, I., \& Gannon, B. (2018). Measurement tools of resource use and quality of life in clinical trials for dementia or cognitive impairment interventions: A systematically conducted narrative review. International Journal of Geriatric Psychiatry, 33(2), e166-e176.

16. Aguirre, E., Kang, S., Hoare, Z., Edwards, R. T., \& Orrell, M. (2016). How does the EQ-5D perform when measuring quality of life in dementia against two other dementia-specific outcome measures? Quality of Life Research, 25, 45-49.
17. Bulamu, N. B., Kaambwa, B., \& Ratcliffe, J. (2015). A systematic review of instruments for measuring outcomes in economic evaluation within aged care. Health and Quality of Life Outcomes, 13(1), 179.

18. Beer, C., Flicker, L., Horner, B., Bretland, N., Scherer, S., Lautenschlager, N. T., et al. (2010). Factors associated with self and informant ratings of the quality of life of people with dementia living in care facilities: A cross sectional study. PLOS ONE, 5(12), e15621.

19. Surr, C. A., Walwyn, R., Lilley-Kelly, A., et al. (2016). Evaluating the effectiveness and cost-effectiveness of Dementia Care Mapping ${ }^{\mathrm{TM}}$ to enable person-centredcare for people with dementia and their carers (DCM-EPIC) in care homes: Study protocol for a randomised controlled trial. Trials, 17, 300.

20. Reisberg, B. (1988). Functional assessment staging (FAST). Psychopharmacology Bulletin, 24(4), 653-659.

21. Herdman, M. G. C., Lloyd, A., Janssen, M., Kind, P., Parkin, D., Bonsel, G., et al. (2011). Development and preliminary testing of the new five-level version of EQ-5D (EQ-5D-5L). Quality of Life Research, 10, 1727-1736.

22. Smith, S. C., Lamping, D. L., Banerjee, S., Harwood, R. H., Foley, B., Smith, P., et al. (2005). Measurement of health-related quality of life for people with dementia: development of a new instrument (DEMQOL) and an evaluation of current methodology. Health Technology Assessment, 9(10), 1-93.

23. Edelman, P., Fulton, B. R., Kuhn, D., \& Chang, C.-H. (2005). A comparison of three methods of measuring dementia-specific quality of life: Perspectives of residents, staff and observers. The Gerontologist, 45, 27-36.

24. Weiner, M. F., Martin-Cook, K., Svetlik, D. A., et al. (2000). The Quality of Life in Late-Stage Dementia (QUALID) Scale. Journal of the American Medical Directors Association, 1, 114-116.

25. Shrive, F. M., Stuart, H., Quan, H., \& Ghali, W. A. (2006). Dealing with missing data in a multi-question depression scale: A comparison of imputation methods. BMC Medical Research Methodology, 6(1), 57.

26. Altman, D. G. (1999). Practical statistics for medical research. New York: Chapman \& Hall/CRC Press.

27. Streiner, D. L. (2003). Starting at the beginning: An introduction to coefficient alpha and internal consistency. Journal of Personality Assessment, 80, 99-103.

28. Hughes, L. J., Farina, N., Page, T. E., Tabet, N., \& Banerjee, S. (2019). Psychometric properties and feasibility of use of dementia specific quality of life instruments for use in care settings: A systematic review. International Psychogeriatrics. https://doi. org/10.1017/S1041610218002259.

29. Sloane, P. D. Z. S., Williams, C. S., Reed, P. S., Gill, K. S., \& Preisser, J. S. (2005). Evaluating the quality of life of long-term care residents with dementia. The Gerontologist, 45(Spec No. 1), 37-49.

30. Moyle, W., Gracia, N., Murfield, J. E., Griffiths, S. G., \& Venturato, L. (2012). Assessing quality of life of older people with dementia in long-term care: A comparison of two self-report measures. Journal of Clinical Nursing, 21, 1632-1640.

31. Robertson, S., Cooper, C., Hoe, J., Lord, K., Rapaport, P., Marston, L., et al. (2019). Comparing proxy rated quality of life of people living with dementia in care homes. Psychological Medicine. https://doi.org/10.1017/S0033291718003987.

32. Landis, J. R., \& Koch, G. G. (1977). The measurement of observer agreement for categorical data. Biometrics, 33(1), 159-174.

33. Orgeta, V., Edwards, R. T., Hounsome, B., Orrell, M., \& Woods, B. (2015). The use of the EQ-5D as a measure of health-related quality of life in people with dementia and their carers. Quality of Life Research, 24(2), 315-324. 
34. Hurt, C. S., Banerjee, C., Tunnard, C., Whitehead, D. L., Tsolaki, M., Mecocci, P., et al. (2010). Insight, cognition and quality of life in Alzheimer's disease. Journal of Neurology, Neurosurgery and Psychiatry, 81(3), 331-336.

35. Clare, L. (2003). Managing threats to self-awareness in early stage Alzheimer's disease. Social Science and Medicine, 57, 1017-1029.

36. Clare, L. (2003). Managing threats to self-awareness in early stage Alzheimer's disease. Social Science and Medicine, 57, 1017-1029.

37. Hughes, L. J., Farina, N., Page, T. E., Tabet, N., \& Banerjee, S. (2019). Psychometric properties and feasibility of use of dementia specific quality of life instruments for use in care settings: A systematic review. International Psychogeriatrics. https://doi. org/10.1017/S1041610218002259.

38. Missotten, P., Dupuis, G., \& Adam, S. (2016). Dementia-specific quality of life instruments: A conceptual analysis. International Psychogeriatrics, 28(8), 1245-1262.

39. Falk, H., Persson, L.-O., \& Wijk, H. (2007). A psychometric evaluation of a Swedish version of the Quality of Life in Late-Stage Dementia (QUALID) scale. International Psychogeriatrics, 19, 1040-1050.
40. Garre-Olmo, J., et al. (2010). Cross-cultural adaptation and psychometric validation of a Spanish version of the Quality of Life in Late-Stage Dementia Scale. Quality of Life Research, 19, 445-453.

41. O’Shea, E., Hopper, L., Marques, M., Gonçalves-Pereira, M., Woods, B., Jelley, H., et al. (2018). A comparison of self and proxy quality of life ratings for people with dementia and their carers: A European prospective cohort study. Aging \& Mental Health. https://doi.org/10.1080/13607863.2018.1517727.

42. Hughes, L. J., Farina, N., Page, T. E., Tabet, N., \& Banerjee, S. (2019). Adaptation of the DEMQOL-Proxy for routine use in care homes: A cross-sectional study of the reliability and validity of DEMQOL-CH. British Medical Journal Open, 9(8), e028045.

Publisher's Note Springer Nature remains neutral with regard to jurisdictional claims in published maps and institutional affiliations. 